\title{
Evaluation of a Crataegus-Based Multiherb Formula for Dyslipidemia: A Randomized, Double-Blind, Placebo-Controlled Clinical Trial
}

\author{
Miao Hu, Weiwei Zeng, and Brian Tomlinson \\ Department of Medicine \& Therapeutics, The Chinese University of Hong Kong, Prince of Wales Hospital, Shatin, Hong Kong \\ Correspondence should be addressed to Brian Tomlinson; btomlinson@cuhk.edu.hk
}

Received 17 December 2013; Revised 19 March 2014; Accepted 22 March 2014; Published 16 April 2014

Academic Editor: Xingjiang Xiong

Copyright (C) 2014 Miao Hu et al. This is an open access article distributed under the Creative Commons Attribution License, which permits unrestricted use, distribution, and reproduction in any medium, provided the original work is properly cited.

\begin{abstract}
Background. We for the first time examined the effects of a multiherb formula containing Crataegus pinnatifida (1g daily), Alisma orientalis, Stigma maydis, Ganoderma lucidum, Polygonum multiflorum, and Morus alba on plasma lipid and glucose levels in Chinese patients with dyslipidemia. Methods. In this randomized, double-blind, placebo-controlled study, 42 patients were randomized at a ratio of $1: 1$ to receive the herbal formula or placebo for 12 weeks and 40 patients completed the study. Lipid profiles, glucose, glycated haemoglobin (HbAlc), and laboratory safety parameters were performed before and after treatment. Results. The difference in the changes in low-density lipoprotein cholesterol (LDL-C) levels between placebo and active treatment (-9\%) was significantly $(P<0.05)$ better with active treatment. HbAlc levels significantly decreased by $-3.9 \%$ in the active treatment group, but the change was not significantly different from that with placebo $(-1.1 \%)(P=0.098)$. There were no apparent adverse effects or changes in laboratory safety parameters with either treatment. Conclusions. The multiherb formula had mild beneficial effects on plasma LDL-C after 12-weeks treatment in subjects with dyslipidemia without any noticeable adverse effects.
\end{abstract}

\section{Introduction}

Herbal medicines have been used for thousands of years in China and other Eastern countries and have regained popularity in Western Countries in the last two decades $[1,2]$. There is growing awareness of the place of dietary factors and herbal medicines in the prevention of cardiovascular disease (CVD) and the possibility of their use in treatment of CVD risk factors [3]. Herbal materials have also resulted in the development of many important conventional drugs, including digoxin and the 3-hydroxy-3-methylglutaryl-coenzyme A reductase inhibitors or statins. More recently, some natural products were found to be effective in the treatment of hyperlipidaemia and diabetes in animal and in human studies [4-6]. Among 57 traditional Chinese medicine (TCM) formulas that have been approved by the China Food and Drug Administration (CFDA) to treat hyperlipidaemia in mainland China, hawthorn fruit (Crataegus pinnatifida Bge.), also known as Shan Zha, is the most popular TCM prescribed in more than $50 \%$ of the formulas, followed by Polygonum multiflorum (or Fo-ti root, known as He Shou Wu in China) (38\%) and Alisma orientalis (Ze Xie) (33\%) [6].

Crataegus products are widely used for the treatment of cardiac and circulatory disorders, particularly for angina, heart failure, and hyperlipidaemia as they are considered to have multiple cardiovascular protective effects (Figure 1) [7-9]. Crataegus leaves, flowers, and fruits contain varying amounts of a number of biologically active substances, such as oligomeric procyanidins, flavonoids, and triterpenes [10, 11]. Among these components, flavonoids and triterpenes, especially ursolic acid, have been reported as the main active constituents exerting hypolipidaemic effects [12]. Recent research indicated that the lipid-lowering effect of Crataegus may be related to the inhibitory effects of flavonoids on 3-hydroxy-3-methylglutaryl-coenzyme A reductase [10], downregulation of intestinal acyl-coA : cholesterol acyltransferase activity by the triterpenes [13], and activation of peroxisome proliferator activated receptor (PPAR) alpha in adipose tissue [14] or in liver [15]. Furthermore, Crataegus extract has been reported to have antioxidant and nitrite 


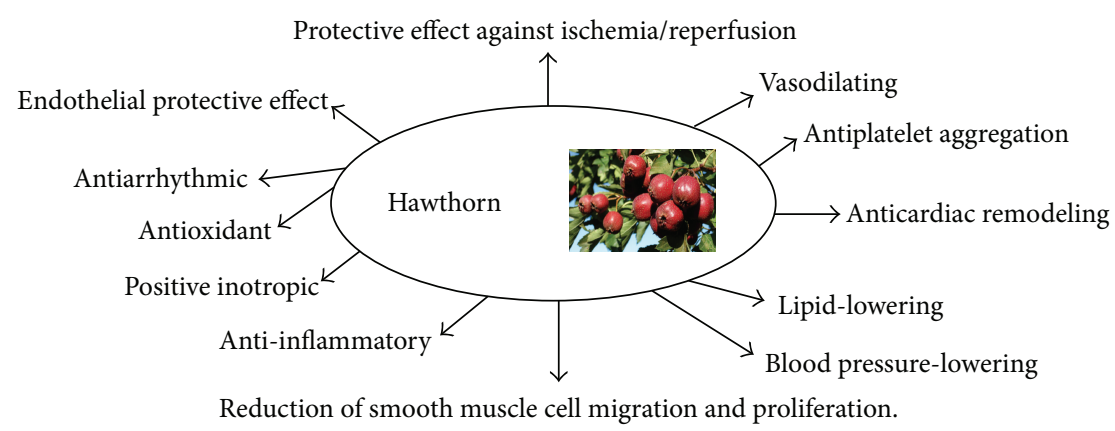

FIGURE 1: Multiple cardiovascular protective effects of Crataegus. Adapted from [9].

reductase activities and inhibits the formation of thromboxane to modify cardiovascular risk factors [16-18]. Although Crataegus has been found to decrease the plasma levels of low-density lipoprotein cholesterol (LDL-C), triglycerides, and glucose in various animal models [14,19-22], limited data are available in humans.

Polygonum multiflorum and Alisma orientalis are also important components in TCM formulas for the treatment of hyperlipidaemia [6]. Stilbene glycosides extracted from the root of Polygonum multiflorum showed antagonistic effects on oxidation of lipoproteins and proliferation of coronary arterial smooth muscle cells and a decrease in their content of nitric oxide [23]. Some animal studies and an early uncontrolled clinical study suggested that Polygonum multiflorum has lipid-lowering effects which may be related to its regulatory effects on the genes involved in cholesterol synthesis and lipoprotein metabolism [6]. Alisma orientalis has been attributed with multiple pharmacological effects, for example, antidiabetic, antihepatitis, and antidiuretic, and it is utilized to treat hyperglycaemia, hyperlipidaemia, and nephritis and for neuroprotection in TCM [24]. It has been reported that triterpenes (alisol M 23-acetate and alisol A 23acetate) from Alisma orientalis act as farnesoid $\mathrm{X}$ receptor agonists, which may be responsible for the antidiabetic and other therapeutic effects of Alisma orientalis [24]. In rats with experimental nonalcoholic fatty liver disease induced by high-fat diet, administration of Alisma orientalis (150, 300 , and $600 \mathrm{mg} / \mathrm{kg}$ ) markedly decreased the serum and liver lipids, decreased plasma glucose levels, improved insulin resistance, and reduced markers of liver injury, aminotransferase abnormalities, and hepatomegaly [25].

Some other herbs such as Ganoderma lucidum (Ling Zhi), Stigma maydis (corn silk or Yu Mi Xu in Chinese), and Morus alba $L$. (Sang Ye) have also been recommended for a long time for protection of CVD, partly through their potential benefits in reducing plasma lipids and glucose levels [2628]. In obese diabetic mice, a water extract of Ganoderma lucidum lowered the plasma LDL-C levels without affecting the expression of 3-hydroxy-3-methylglutaryl-coenzyme A reductase in both hepatic and extrahepatic organs [29]. Double-blinded, placebo-controlled, and crossover intervention studies in healthy volunteers and in patients with diabetes showed that Ganoderma lucidum supplement (1.44 g daily) may improve plasma lipid profiles [30, 31].
Stigma maydis is rich in phenolic compounds, particularly flavonoids [28]. A recent animal study showed that the flavonoids from Stigma maydis extract significantly lowered plasma levels of LDL-C and triglycerides in rats fed highcholesterol diet indicating that flavonoids from Stigma maydis extract may have potential antihyperlipidemic effects [32]. Morus alba L. has been used in traditional Chinese medicine for cardiovascular, liver, and spleen disorders. Several animal studies in rats have demonstrated that Morus alba $L$. reduced plasma triglycerides, free fatty acid, and/or LDL-C $[33,34]$. DNA microarray analysis revealed that mulberry leaves upregulated expression of the genes involved in the peroxisome proliferator-activated receptor signaling pathway and downregulated the genes involved in lipogenesis [33]. In another study in hamsters fed with high fat/cholesterol diets, Morus alba L. extract (1\% and 2\%) significantly reduced plasma total cholesterol and triglyceride levels by $30-37 \%$ and 16-35\%, respectively [35]. Low-density lipoprotein receptor gene expression and the uptake ability of LDL in HepG2 cells were upregulated, whereas the gene expressions of enzymes involved in triglyceride and cholesterol biosyntheses were decreased with Morus alba L. [35]. These studies suggested that Stigma maydis and Morus alba L. can be used as natural agents against hyperlipidaemia. However, to the best of our knowledge, there is no study reporting the effect of these natural products on plasma lipid profiles in human.

The present study for the first time examined the effect of a commercially available multiherb formula containing the herbs mentioned above (Table 1 ) on reducing plasma lipid and glucose levels in Chinese patients with dyslipidemia.

\section{Materials and Methods}

\subsection{Participants}

2.1.1. Inclusion Criteria. Subjects aged $\geq 18$ years with dyslipidemia (familial or nonfamilial) were recruited from the patients who were regularly attending the Lipid Clinic in the Prince of Wales Hospital, Hong Kong. Dyslipidemia was defined as either having a documented history of dyslipidemia and receiving lipid-lowering therapy or having a documented elevated baseline fasting LDL-C cholesterol ( $\geq 4.1 \mathrm{mmol} / \mathrm{L}$ ) or triglycerides $\geq 1.7 \mathrm{mmol} / \mathrm{L}$ based on local laboratory reference values. Patients were eligible if they had 
TABLE 1: The multiherb formula tested in the study.

\begin{tabular}{lcc}
\hline $\begin{array}{l}\text { Herb extracts } \\
\text { (Chinese pinyin names) }\end{array}$ & $\begin{array}{c}\text { Weight } \\
\text { (mg per capsule) }\end{array}$ & Proportions \\
\hline $\begin{array}{l}\text { Crataegus pinnatifida } \\
\text { (Shan Zha) }\end{array}$ & 129 & $30 \%$ \\
$\begin{array}{l}\text { Alisma orientalis } \\
\text { (Ze Xie) }\end{array}$ & 86 & $20 \%$ \\
$\begin{array}{l}\text { Stigma maydis } \\
\text { (Yu Mi Xu) }\end{array}$ & 86 & $20 \%$ \\
$\begin{array}{l}\text { Ganoderma lucidum } \\
\text { (Ling Zhi) }\end{array}$ & 43 & $10 \%$ \\
$\begin{array}{l}\text { Polygonum multiflorum } \\
\text { (He Shou Wu) }\end{array}$ & 43 & $10 \%$ \\
$\begin{array}{l}\text { Marus alba } \\
\text { (Sang Ye) }\end{array}$ & 43 & $10 \%$ \\
\hline
\end{tabular}

a plasma level of LDL-C $\geq 2.6 \mathrm{mmol} / \mathrm{L}$ or $\geq 1.8 \mathrm{mmol} / \mathrm{L}$ for those with high cardiovascular risk (because of a history of coronary heart disease (CHD), other clinical evidence of atherosclerosis, diabetes mellitus, or calculated 10-year CHD risk score $>20 \%$ ) following advice on a lipid-lowering diet with lipid-lowering treatment or if they had elevated plasma triglyceride concentrations $(\geq 1.7 \mathrm{mmol} / \mathrm{L})$ following advice on a lipid-lowering diet with or without lipid-lowering treatment.

2.1.2. Exclusion Criteria. Exclusion criteria included a history of myocardial infarction, stroke, coronary artery bypass surgery or other revascularization procedures, unstable angina, or angioplasty within 3 months of screening; elevated liver enzymes (alanine aminotransferase $[\mathrm{ALT}]>1.5 \times \mathrm{ULN}$ ) or renal impairment (plasma creatinine $>200 \mu \mathrm{mol} / \mathrm{L}$ ) or uncontrolled endocrine or metabolic disease known to influence serum lipids or lipoproteins; a history of alcohol or drug abuse; a history of hypersensitivity to any of the ingredients contained in the study herb formula; women who were pregnant or lactating; initiation of lipid-lowering therapy or antidiabetic treatment within 4 weeks prior to screening. Subjects were excluded if they took weight lowering agents with 6 months prior to screening or were currently engaging in vigorous exercise or aggressive diet regimens for weight control.

The study protocol and statement of informed consent were approved by the Joint Clinical Research Ethics Committee of The Chinese University of Hong Kong and New Territories East Cluster before the start of the study. The study was conducted in compliance with the Declaration of Helsinki and all participants gave written informed consent.

2.2. Study Medication. The herbal formula (blood fat droplets (control)) and matching placebo were manufactured and supplied by Vita Green Pharmaceutical (HK) Ltd (Hong Kong, China). This product is registered for use as a natural health product in Hong Kong. Pretreated Crataegus pinnatifida, Alisma orientalis, Polygonum multiflorum, Ganoderma lucidum, and Stigma maydis were extracted with water : ethanol $(1: 1)$ at $60^{\circ} \mathrm{C}$ and then extracted again with water alone. The extract was then concentrated. Morus alba was extracted with water at $80^{\circ} \mathrm{C}$ and then concentrated. The extracts of Crataegus pinnatifida, Alisma orientalis, Stigma maydis, Polygonum multiflorum, Ganoderma lucidum, and Morus alba were mixed (in a ratio of $3: 2: 2: 1: 1: 1$ ), vacuum dried, and ground into powder. The dosages of each herb in the final herb formula are shown in Table 1.

Ursolic acid was used as the quality marker of this herbal product and was quantified by HPLC. The mobile phase contained methanol-water-glacial acetic acid $(88: 12: 0.2)$. UV detection was performed at $215 \mathrm{~nm}$. The sample was defatted with petroleum ether and extracted using diethyl ether. The solvent was evaporated and the residue dissolved in methanol for injection. The specification is $>0.01 \%$ ursolic acid in the final product. Heavy metal and toxic elements including arsenic, lead, and mercury were measured using in house methods. Microbial examination (total aerobic count, moulds and yeast count, and Escherichia coli) and pesticides residue analysis were also performed on the samples.

The placebo capsule contained starch and artificial food colouring. The herb formula and placebo were identical in packing, appearance, and colour.

2.3. Study Protocol. This study was a prospective, randomized, double-blinded, placebo-controlled, and parallel design study. After completion of a 2 -week placebo run-in period, eligible patients were randomly assigned to receive the herbal formula or placebo which was consumed twice daily with or without food, four capsules in the morning and four capsules in the evening for a period of 12 weeks.

Randomization was performed using Random Allocation Software (Version 1.0, Isfahan University of Medical Sciences, Isfahan, Iran) that allows random lists to be generated with permuted block and designated seeds. Electronic and paper records of the randomization seed number and the randomization sequence were kept in the study center for operation. The allocation sequence was generated by Miao $\mathrm{Hu}$ (the first author), whereas participant enrollment and assignment were conducted by Brian Tomlinson and the study nurses. The patients, investigators, and the study staff were blinded to treatment assignment until the outcome assessment was completed.

Patients were assessed at baseline and at 6 and 12 weeks after the initiation of treatment with herb formula or placebo, with the last dose being consumed the evening before the visits. Anthropometric measurements, including body weight, waist circumference, hip circumference, and estimation of percentage body fat using an impedance device (TANITA Body Composition Analyzer BF-350, Tokyo, Japan), were performed by a research nurse. Blood pressure was measured by a semiautomatic sphygmomanometer (Critikon Dinamap; GE Medical Systems Information Technologies, Louisville, KY, USA). Fasting blood samples were taken for lipid profile, fasting glucose, and laboratory safety tests at the study visit. Adherence to study medication and tolerability were assessed at study visits. All subjects were asked to maintain their usual diet and other aspects of lifestyle during the study. 
2.4. Sample Size Estimation. Some previous randomized, placebo-controlled studies with Crataegus pinnatifida or other herbs showed that a minimal number of 10-30 patients in each group were needed to demonstrate the significant effect of herb supplements on lipid and glucose levels depending on the effect of the herbs $[30,31,36]$, but clinical data on the lipid-lowering effect of this herb product or with other similar herbal formulas in patients with dyslipidemia is still lacking. Assuming that this herb formula would show a mean of $20 \%$ greater reduction in LDL-C than placebo with the SD of the \% reduction in LDL-C being $4 \%$ as some animal studies showed that some of these herbs might decrease plasma lipids by up to over $30 \%$ [35], 16 subjects in each group would be needed for $80 \%$ power with $\alpha=0.05$. Considering a drop-out rate of $20 \%$, we planned to recruit at least 40 patients with dyslipidemia in the present study.

2.5. Biochemistry Measurements. All biochemistry tests including lipid profiles, glucose, glycated haemoglobin (HbAlc), and laboratory safety parameters (e.g., creatinine, creatine kinase, total bilirubin, alkaline phosphatase (ALP), and ALT) were performed by standard methods in the Chemical Pathology laboratory at the Prince of Wales Hospital, which has international laboratory accreditation. Total cholesterol level was measured by the enzymatic method (Centrichem Chemistry System, Baker Instruments Co. Allentown). High-density lipoprotein cholesterol (HDLC) level was determined by using the fractional precipitation of dextran sulphate with manganous ion. Triglyceride levels were measured by the glyceryl dehydrogenase reaction following the hydrolysis of the triglyceride (Centrichem Chemistry System, Baker Instruments Co., Allentown). LDL-C concentrations were calculated according to the Friedewald formula or directly measured if the triglyceride level was greater than $4.5 \mathrm{mmol} / \mathrm{L}$ [37].

2.6. Statistical Analysis. Per protocol analysis was performed in a blind manner in 40 patients who had completed all study visits. The primary end point of the study was percentage change in LDL-C from baseline at 12 weeks. The secondary end points included percentage changes in other lipid parameters, HbAlc, fasting plasma glucose, and laboratory safety tests at 6 and 12 weeks. Continuous variables were expressed as mean \pm SD unless otherwise indicated. Skewed data were logarithmically transformed before analysis. The baseline characteristics and the primary and secondary outcomes between the two treatment groups were compared using Student's $t$-test for normally distributed parameters or MannWhitney test for continuous variables that could not be successfully transformed into normally distributed data and chi-square tests for categorical variables. Paired-sample $t$ test was performed to assess changes of parameters within the placebo or active treatment group. Differences were considered to be statistically significant, if the two-sided $P$ value is $<0.05$. Statistical analysis was performed using the Statistical Package for the Social Sciences version 17.0 (SPSS Inc., Chicago, IL, USA).
TABLE 2: Baseline characteristics of the study participants.

\begin{tabular}{lcc}
\hline & $\begin{array}{c}\text { Placebo } \\
(n=20)\end{array}$ & $\begin{array}{c}\text { Herb formula } \\
(n=20)\end{array}$ \\
\hline Age, years & $54.7 \pm 9.1$ & $57.5 \pm 8.4$ \\
Males, $n$ (\%) & $10(50)$ & $6(30)$ \\
Body weight, kg & $71.3 \pm 17.2$ & $65.0 \pm 10.7$ \\
Body mass index, kg/m ${ }^{2}$ & $27.2 \pm 4.7$ & $25.9 \pm 2.8$ \\
Body fat, \% & $31.5 \pm 7.3$ & $31.9 \pm 6.1$ \\
Waist, cm & $94.3 \pm 12.7$ & $87.7 \pm 7.7$ \\
SBP, mmHg & $119.5 \pm 12.4$ & $118.9 \pm 10.8$ \\
DBP, mmHg & $77.1 \pm 9.6$ & $73.6 \pm 9.6$ \\
Pulse, bpm & $71.1 \pm 10.1$ & $67.4 \pm 7.5$ \\
Diabetes, $n$ (\%) & $8(40)$ & $11(55)$ \\
Hypertension, $n$ (\%) & $14(70)$ & $10(50)$ \\
FH, $n$ (\%) & $7(35)$ & $5(25)$ \\
On lipid-lowering treatment & $9(45)$ & $8(40)$ \\
On antidiabetic treatment & $5(25)$ & $5(25)$ \\
Baseline TC, mmol/L & $5.39 \pm 0.89$ & $5.90 \pm 1.17$ \\
Baseline HDL-C, mmol/L & $1.29 \pm 0.39$ & $1.28 \pm 0.25$ \\
Baseline TG, mmol/L & $2.35 \pm 1.18$ & $2.00 \pm 0.71$ \\
Baseline LDL-C, mmol/L & $3.04 \pm 0.82$ & $3.72 \pm 1.17^{*}$ \\
Baseline non-HDL-C, mmol/L & $4.10 \pm 0.68$ & $4.63 \pm 1.13$ \\
Fasting glucose, mmol/L & $5.57 \pm 1.23$ & $5.84 \pm 2.17$ \\
HbAlc, \% & $6.57 \pm 0.98$ & $6.59 \pm 0.83$ \\
\hline DBP: & & \\
\hline
\end{tabular}

DBP: diastolic blood pressure; TC: total cholesterol; FH: familial hypercholesterolaemia; HDL-C: high-density lipoprotein cholesterol; LDL-C: lowdensity lipoprotein cholesterol; SBP: systolic blood pressure; TG: triglycerides.

${ }^{*} P<0.05$ versus placebo.

\section{Results}

3.1. Characteristics of Study Participants. A total of 43 patients were recruited for the study and 1 patient withdrew consent. A total of 42 patients were randomized and two patients dropped out due to adverse effects (one in each group) (Figure 2). Forty patients completed all study-related visits. Of those patients who completed the study, 16 (40\%) were male, 19 (47.5\%) had type 2 diabetes, and 12 (30\%) had familial hypercholesterolemia (Table 2$)$. The mean $( \pm$ SD) age was $56 \pm 8.8$ years and the body mass index was $26.5 \pm$ $3.9 \mathrm{~kg} / \mathrm{m}^{2}$. Seventeen patients were on a background of stable lipid-lowering treatment with 14 of them receiving statins and 3 patients receiving gemfibrozil. There were no statistically significant differences in the baseline characteristics between the two groups except that the baseline LDL-C levels were higher in the active treatment groups than those in the placebo group $(3.72 \pm 1.17$ versus $3.04 \pm 0.82 \mathrm{mmol} / \mathrm{L}$, $P<0.05)$.

3.2. Effects on Lipids and Glucose Levels. There were no significant changes in body weight between baseline and after treatment for each of the groups during the study (Table 3). The baseline LDL-C levels were not associated with 


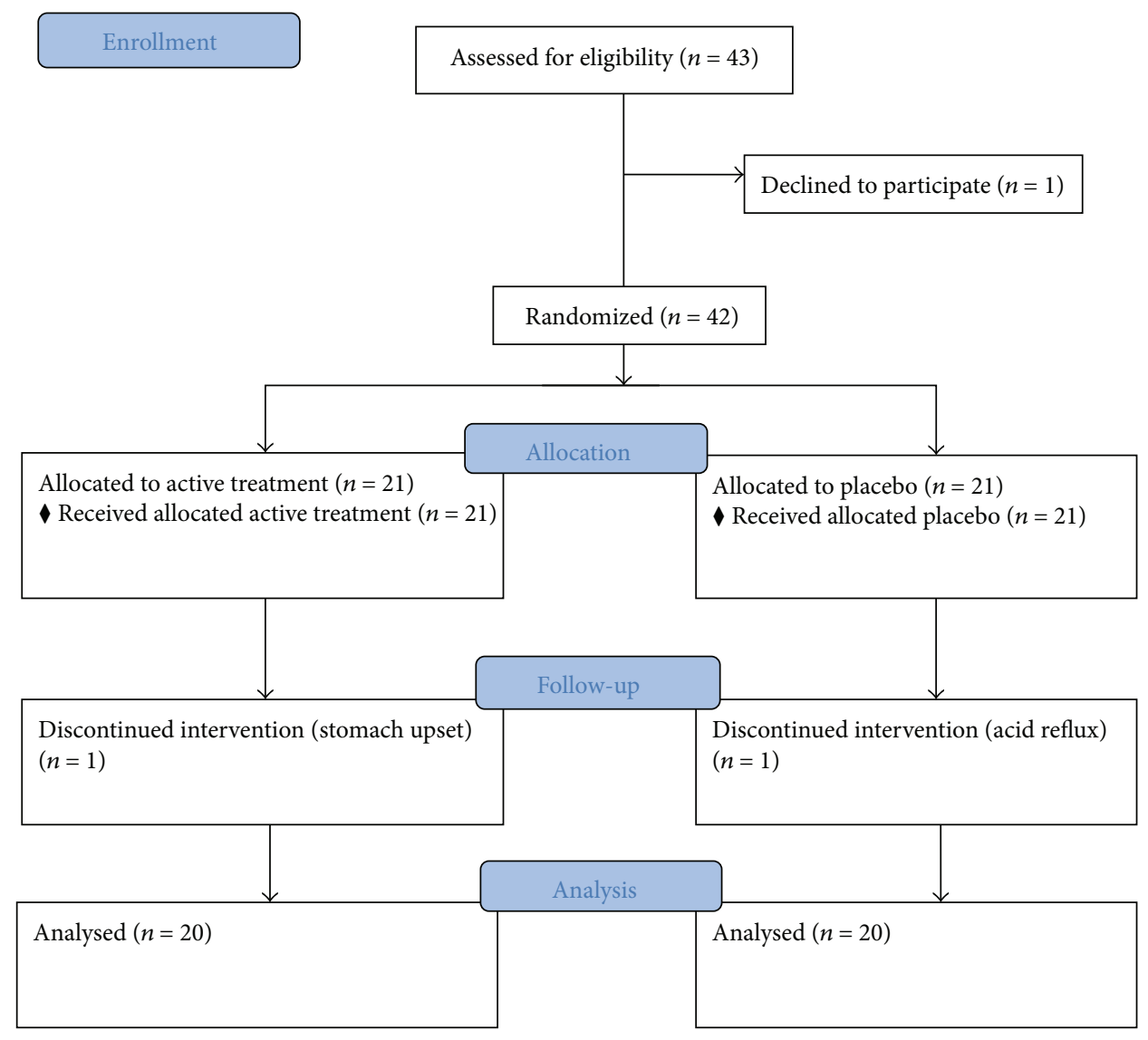

FIGURE 2: CONSORT flowchart of study recruitment and completion of the study.

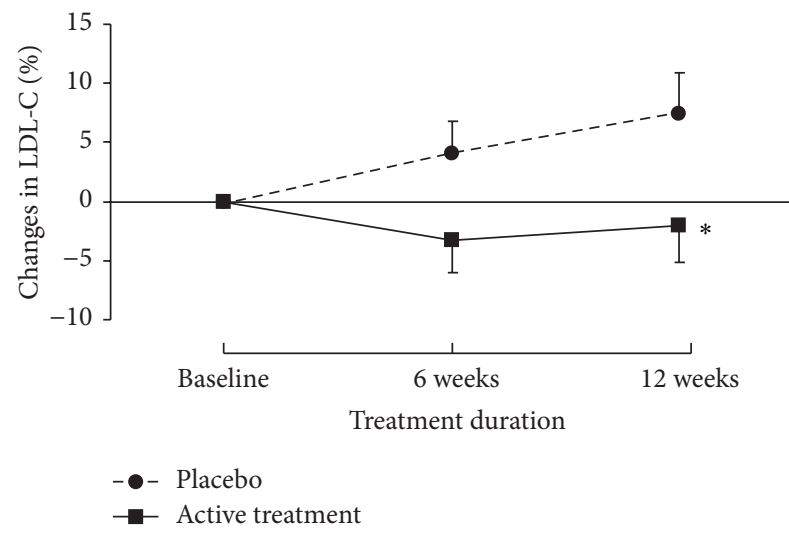

Figure 3: The percentage changes from baseline in LDL-C after 6 and 12 weeks of treatment. ${ }^{*} P<0.05$.

the LDL-C response to the active treatment and placebo (data not shown). After 6 and 12 weeks of treatment, the LDL$\mathrm{C}$ levels increased by $4 \%$ and $7.4 \%$ in the placebo group, while they decreased by $-3 \%$ and $-2 \%$ in the active treatment group, and the difference in changes in LDL-C at week 12 between placebo and active treatment groups was significant $(P=0.062$ and $P<0.05$ versus placebo, after 6 and 12 weeks,

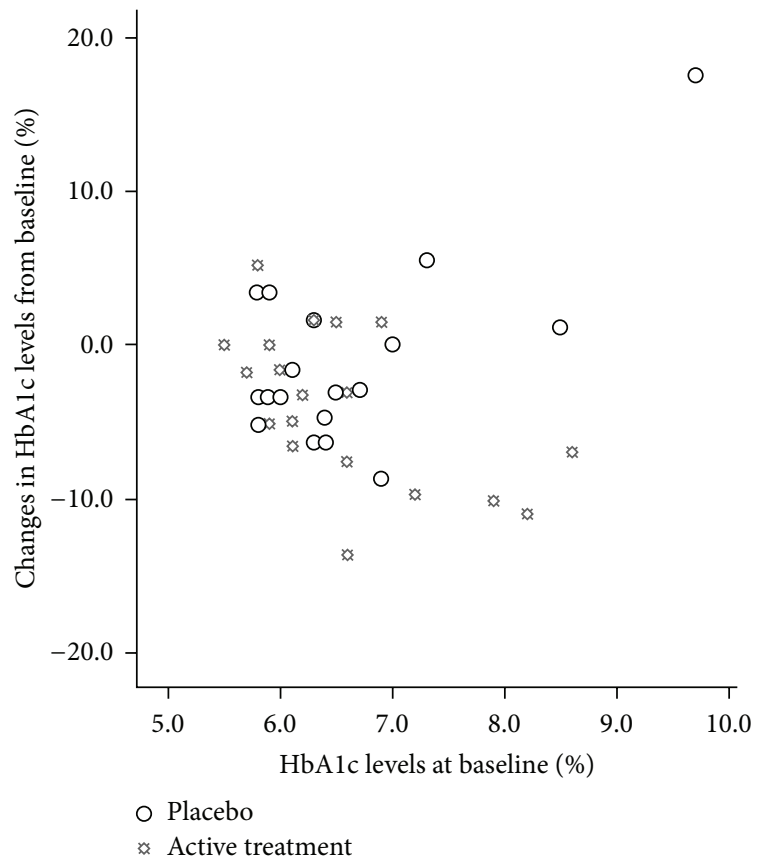

Figure 4: Association between the baseline levels of glycated haemoglobin and the percentage changes in glycated haemoglobin after 12 weeks of treatment. 
resp.) (Figure 3). The mean level of triglycerides tended to be decreased in the placebo group and increased in the active treatment after 12 weeks of therapy, but this change was not statistically different compared to baseline or compared to the alternative regimen. The increase in the active treatment group was largely driven by an outlier who had an increase of triglycerides from $2.3 \mathrm{mmol} / \mathrm{L}$ to $8.5 \mathrm{mmol} / \mathrm{L}$ at the end of the study. His triglycerides after 6-week treatment with the herb product was $2.0 \mathrm{mmol} / \mathrm{L}$ suggesting that the increase in triglycerides at the end of study is likely related to changes in diet or other aspects of lifestyle which occurred during the longer follow-up period.

There was no significant difference in the changes in other lipid parameters and fasting plasma glucose between the two groups (Table 3 ). The HbAlc levels significantly decreased by $-3.9 \%$ in the active treatment group after 12 weeks of treatment, but this was not significantly different from the change with placebo $(-1.1 \%)(P=0.098)$. There was an inverse correlation between baseline HbAlc levels and the changes in HbAlc in the active treatment group $(r=$ $-0.565, P=0.01$ ), so that patients with higher baseline levels tended to show a greater fall, but this was not seen in the placebo group with or without including one outlier $(P>0.05)$ (Figure 4). Background lipid-lowering treatment or antidiabetic treatment had no effect on the lipid and glucose response to the treatment (data not shown).

3.3. Adverse Effects. Both active treatment and placebo were well tolerated. Plasma biochemical parameters in the two groups are shown in Table 4 . All the laboratory safety parameters were within the normal range during the study. The plasma urea level in the placebo group was increased by $10.2 \%$, which was significantly different from the decrease of $-3.6 \%$ in the active treatment group $(P<0.05)$. There was a significant increase in the plasma albumin levels in the active treatment group but no difference in the changes in albumin levels between the two groups.

A total of 28 adverse events were reported by 23 participants (12 in the placebo group and 11 in the active treatment group). Of the two patients who withdrew from the study, one patient randomized to the active treatment complained of stomach upset and one patient randomized to placebo developed acid reflux. Influenza and cough were the most common adverse events $(n=8)$ followed by shoulder or knee pain $(n=5)$ and headache $(n=3)$. None of the adverse events were considered clinically significant. There was no statistically significant difference in rates of any adverse events among the treatment groups.

\section{Discussion}

In this randomized clinical study of a multiherb product containing Crataegus pinnatifida, Alisma orientalis, Stigma maydis, and other herbs which have long been considered to have hypolipidaemic and/or hypoglycaemic effects, there was a significant improvement in plasma LDL-C levels in patients receiving the active treatment for 12 weeks compared to those assigned to placebo (difference 9.4\%) and this trend was already observed at 6 weeks. However, the change in LDL-C in the active treatment group is very small and the difference in the LDL-C response in the two groups may be largely driven by the increase in the LDL-C levels in the placebo group and may be influenced by the difference in baseline LDL-C levels.

It was shown that hawthorn fruit drink $250 \mathrm{~mL}$ (containing $1.4 \mathrm{mg}$ hawthorn flavones) twice daily significantly decreased the plasma LDL-C, apolipoprotein B, and triglycerides by $10.4 \%, 7.4 \%$, and $9.3 \%$, respectively, in 30 Chinese patients with dyslipidemia in an early uncontrolled study [22], although another placebo-controlled study showed a Chinese therapeutic food supplement with hawthorn fruit and Chinese kiwifruit-extract compound had no effect on plasma LDL-C or triglyceride levels but it increased the HDL-C by $5 \%$ in Caucasian patients with dyslipidemia [38]. Several factors may contribute to the limited lipid-lowering effect of the tested herbal product in the present study. Insufficient dosing is one of the possibilities. Furthermore, the relatively mild elevated baseline plasma levels of LDL-C and background of lipid-lowering drugs may also influence the lipid-lowering efficacy although there was no significant association between the baseline LDL-C level, the baseline lipid-lowering treatment, and the lipid responses to the herb product. Another potential confounder is that our study recruited a rather heterogeneous group of patients receiving different treatments for their comorbidities, and this may contribute to the variability of the lipid response to treatment and limit the power of the study to detect a significant effect of the supplement. However, herbal supplements are often used concomitantly with conventional drugs, especially in the elderly or those with chronic disease such as dyslipidemia and diabetes, and the goal of the study was to evaluate the realworld effect of the herbal product.

There was no statistically significant difference in the percentage change in $\mathrm{HbAlc}$ levels between the herb formula and placebo during the study, although there was a significant decrease in HbAlc levels in the active treatment group, particularly in patients with higher baseline levels, which is a typical finding with many antihyperglycaemic drugs. This result may suggest a potential beneficial effect of this supplement on the overall glycaemic control in patients with abnormal metabolic states of glucose regulation, for example, impaired glucose tolerance and diabetes. Several lines of evidence suggest the effectiveness of Crataegus pinnatifida, Alisma orientalis, and other herbs contained in this herb supplement on glucose and lipid metabolism [5, 6, 12, 24]. Flavonoids and triterpenes appear to be the main active components of these herbs to exert antihyperlipidaemia and antihyperglycaemia effect. However, this study was not designed to examine the effect of this herb formula in treating diabetes. Further research with larger sample size is needed to investigate the hypoglycaemic effect of this supplement in patients with diabetes.

This study has several limitations which need to be considered. Firstly, there was significant difference in the baseline LDL-C levels between the placebo group and the active treatment group. This may be related to the small sample size. In this study, patients were selected if they had 
TABLE 3: Changes in body weight, lipids, and glucose at week 12.

\begin{tabular}{|c|c|c|c|c|c|c|c|}
\hline & \multicolumn{3}{|c|}{ Placebo $(n=20)$} & \multicolumn{3}{|c|}{ Herb formula $(n=20)$} & \multirow{2}{*}{$\begin{array}{c}P \text { value } \\
\text { (versus placebo) }\end{array}$} \\
\hline & Baseline & Week 12 & $\%$ change & Baseline & Week 12 & $\%$ change & \\
\hline Body weight, kg & $71.3 \pm 17.2$ & $69.0 \pm 20.9$ & $-2.7 \pm 15.0$ & $65.0 \pm 10.7$ & $65.7 \pm 10.0$ & $0.8 \pm 1.9$ & 0.310 \\
\hline $\mathrm{TC}, \mathrm{mmol} / \mathrm{L}$ & $5.39 \pm 0.89$ & $5.61 \pm 1.01$ & $4.5 \pm 10.3$ & $5.90 \pm 1.17$ & $5.96 \pm 1.21$ & $1.4 \pm 10.5$ & 0.362 \\
\hline $\mathrm{HDL}-\mathrm{C}, \mathrm{mmol} / \mathrm{L}$ & $1.29 \pm 0.39$ & $1.34 \pm 0.46$ & $0.7 \pm 12.4$ & $1.28 \pm 0.25$ & $1.27 \pm 0.30$ & $2.0 \pm 12.4$ & 0.276 \\
\hline $\mathrm{TG}, \mathrm{mmol} / \mathrm{L}$ & $2.35 \pm 1.18$ & $2.21 \pm 1.04$ & $-6.7(-18.3,15.8)$ & $2.00 \pm 0.71$ & $2.42 \pm 1.61$ & $5.8(-14.8,16.3)$ & 0.383 \\
\hline LDL-C, $\mathrm{mmol} / \mathrm{L}$ & $3.04 \pm 0.82$ & $3.27 \pm 0.95^{*}$ & $7.4 \pm 15.5$ & $3.72 \pm 1.17$ & $3.64 \pm 1.17$ & $-2.0 \pm 13.6$ & 0.049 \\
\hline Non-HDL-C, $\mathrm{mmol} / \mathrm{L}$ & $4.10 \pm 0.68$ & $4.28 \pm 0.78$ & $3.6 \pm 9.2$ & $4.63 \pm 1.13$ & $4.69 \pm 1.16$ & $0.4 \pm 12.5$ & 0.368 \\
\hline Fasting glucose, $\mathrm{mmol} / \mathrm{L}$ & $5.57 \pm 1.23$ & $5.80 \pm 2.02$ & $2.9 \pm 12.1$ & $5.84 \pm 2.17$ & $5.80 \pm 1.19$ & $0.3 \pm 7.8$ & 0.432 \\
\hline HbAlc, $\%$ & $6.57 \pm 0.98$ & $6.54 \pm 1.36$ & $-1.1 \pm 5.7$ & $6.59 \pm 0.83$ & $6.31 \pm 0.65^{* *}$ & $-3.9 \pm 4.9$ & 0.098 \\
\hline
\end{tabular}

TC: total cholesterol; HDL-C: high-density lipoprotein cholesterol; LDL-C: low-density lipoprotein cholesterol; TG: triglycerides. Data are presented as mean \pm SD or median (interquartile range).

${ }^{*} P<0.05 ;{ }^{* *} P<0.01$ compared to baseline.

TABLE 4: Changes in biochemical parameters at week 12.

\begin{tabular}{|c|c|c|c|c|c|c|c|}
\hline & \multicolumn{3}{|c|}{ Placebo $(n=20)$} & \multicolumn{3}{|c|}{ Herb formula $(n=20)$} & \multirow{2}{*}{$\begin{array}{c}P \text { value } \\
\text { (versus placebo) }\end{array}$} \\
\hline & Baseline & Week 12 & $\%$ change & Baseline & Week 12 & $\%$ change & \\
\hline Creatinine, $\mu \mathrm{mol} / \mathrm{L}$ & $70.5 \pm 13.1$ & $68.1 \pm 14.2$ & $-3.3 \pm 9.5$ & $68.7 \pm 12.5$ & $66.7 \pm 12.6$ & $-2.5 \pm 9.9$ & 0.799 \\
\hline Creatine kinase, U/L & $144.3 \pm 72.8$ & $135.7 \pm 78.7$ & $-1.0(-26.8,10.1)$ & $144.3 \pm 101.4$ & $141.2 \pm 73.6$ & $1.2(-7.5,15.0)$ & 0.563 \\
\hline Total protein, g/L & $75.2 \pm 3.3$ & $76.1 \pm 3.1$ & $1.3 \pm 3.7$ & $74.4 \pm 4.2$ & $75.1 \pm 3.3$ & $1.1 \pm 3.8$ & 0.860 \\
\hline Urea, mmol/L & $4.78 \pm 1.18$ & $5.13 \pm 1.15$ & $10.2 \pm 24.6$ & $5.35 \pm 1.29$ & $5.13 \pm 1.36$ & $-3.6 \pm 15.6$ & 0.041 \\
\hline Albumin, g/L & $44.9 \pm 2.4$ & $45.3 \pm 2.2$ & $0.9 \pm 4.5$ & $43.5 \pm 2.7$ & $45.0 \pm 2.6^{* *}$ & $3.6 \pm 5.4$ & 0.092 \\
\hline Bilirubin, $\mu \mathrm{mol} / \mathrm{L}$ & $12.6 \pm 5.5$ & $13.1 \pm 6.8$ & $4.9 \pm 25.2$ & $12.1 \pm 4.0$ & $11.9 \pm 5.2$ & $1.4 \pm 32.2$ & 0.706 \\
\hline ALT, IU/L & $31.4 \pm 9.9$ & $32.1 \pm 14.3$ & $3.2 \pm 26.1$ & $24.7 \pm 9.8$ & $26.6 \pm 10.0$ & $17.7 \pm 60.5$ & 0.332 \\
\hline ALP, U/L & $73.8 \pm 15.8$ & $73.9 \pm 14.2$ & $0.8 \pm 7.5$ & $59.5 \pm 16.8$ & $60.0 \pm 14.4$ & $2.1 \pm 11.5$ & 0.672 \\
\hline Urate, $\mathrm{mmol} / \mathrm{L}$ & $0.38 \pm 0.09$ & $0.38 \pm 0.08$ & $3.1 \pm 12.6$ & $0.34 \pm 0.06$ & $0.35 \pm 0.07$ & $0.8 \pm 13.2$ & 0.567 \\
\hline
\end{tabular}

ALT: alanine aminotransferase; ALP: alkaline phosphatase; data are presented as mean $\pm \mathrm{SD}$ or median (interquartile range); ${ }^{* *} P<0.01$ compared to baseline.

either elevated LDL-C and/or elevated triglycerides, and thus they may have different types of dyslipidemia, contributing to the different lipid profiles of the two groups in this small study. The small sample size is another major limitation of the study. The studied herb formula only showed a marginal effect on plasma LDL-C levels compared to placebo and the study is underpowered to detect small differences between the two groups. In addition, it would be useful to measure various apolipoprotein levels, for example, apoAI, apoB, and apoE, although these apolipoprotein levels are usually closely associated with particular plasma lipid levels. It would be helpful to identify and quantify the active components in this herbal formula and to test whether these active components reduce the plasma lipids in a dose-dependent manner, but the study was designed to examine the real-world lipidlowering effect of this commercially available herbal product in patients with dyslipidemia using a recommended dose, and thus the dose-response was not assessed.

In conclusion, this randomized, placebo-controlled study conducted in an ambulatory outpatient setting showed a marginal beneficial effect of the multiherb formula on reducing plasma LDL-C levels in subjects with dyslipidemia without any noticeable adverse effects. The finding was consistent with some of the previous experimental in vitro and animal studies with these herbs. Further well-designed clinical studies are warranted to support or refute the clinical use of herbal medicines in reducing cardiovascular risk.

\section{Conflict of Interests}

The authors have declared that there is no conflict of interests.

\section{Acknowledgments}

The study was partly supported by an unrestricted grant from Vita Green Pharmaceutical (HK) Ltd. This funding source had no role in the study design, the collection, analysis, or interpretation of data or in the writing of the paper. The herbal product and placebo were provided free of charge by Vita Green Pharmaceutical (HK) Ltd, Hong Kong. The authors thank the members of the research team who contributed to this project, especially Ms. Maybo Lin and Ms. Evelyn Chau for their excellent assistance in recruiting and monitoring of patients. 


\section{References}

[1] A. Tachjian, V. Maria, and A. Jahangir, "Use of herbal products and potential interactions in patients with cardiovascular diseases," Journal of the American College of Cardiology, vol. 55, no. 6, pp. 515-525, 2010.

[2] P. S. Fasinu, P. J. Bouic, and B. Rosenkranz, "An overview of the evidence and mechanisms of herb-drug interactions," Frontiers in Pharmacology, vol. 3, article 69, 2012.

[3] Y. L. Yang, S. W. Chan, M. Hu, R. Walden, and B. Tomlinson, "Effects of some common food constituents on cardiovascular disease," ISRN Cardiology, vol. 2011, Article ID 397136, 16 pages, 2011.

[4] S. Hasani-Ranjbar, N. Nayebi, L. Moradi, A. Mehri, B. Larijani, and M. Abdollahi, "The efficacy and safety of herbal medicines used in the treatment of hyperlipidemia; a systematic review," Current Pharmaceutical Design, vol. 16, no. 26, pp. 2935-2947, 2010.

[5] T.-T. Zhang and J.-G. Jiang, "Active ingredients of traditional Chinese medicine in the treatment of diabetes and diabetic complications," Expert Opinion on Investigational Drugs, vol. 21, no. 11, pp. 1625-1642, 2012.

[6] W. Xie, Y. Zhao, and L. Du, "Emerging approaches of traditional Chinese medicine formulas for the treatment of hyperlipidemia," Journal of Ethnopharmacology, vol. 140, no. 2, pp. 345367, 2012.

[7] Q. Chang, Z. Zuo, F. Harrison, and M. S. S. Chow, "Hawthorn," The Journal of Clinical Pharmacology, vol. 42, no. 6, pp. 605-612, 2002.

[8] J. Wu, W. Peng, R. Qin, and H. Zhou, "Crataegus pinnatifida: chemical constituents, pharmacology, and potential applications," Molecules, vol. 19, no. 2, pp. 1685-1712, 2014.

[9] J. Wang, X. Xiong, and B. Feng, "Effect of Crataegus usage in cardiovascular disease prevention: an evidence-based approach," Evidence-Based Complementary and Alternative Medicine, vol. 2013, Article ID 149363, 16 pages, 2013.

[10] X.-L. Ye, W.-W. Huang, Z. Chen et al., "Synergetic effect and structure-activity relationship of 3-hydroxy-3-methylglutaryl coenzyme a reductase inhibitors from crataegus pinnatifida bge," Journal of Agricultural and Food Chemistry, vol. 58, no. 5, pp. 3132-3138, 2010.

[11] J. E. Edwards, P. N. Brown, N. Talent, T. A. Dickinson, and P. R. Shipley, "A review of the chemistry of the genus Crataegus," Phytochemistry, vol. 79, pp. 5-26, 2012.

[12] S.-W. Min, S.-H. Jung, K.-H. Cho, and D.-H. Kim, "Antihyperlipidemic effects of red ginseng, crataegii fructus and their main constituents ginsenoside Rg3 and ursolic acid in mice," Biomolecules \& Therapeutics, vol. 16, no. 4, pp. 364-369, 2008.

[13] Y. Lin, M. A. Vermeer, and E. A. Trautwein, "Triterpenic acids present in hawthorn lower plasma cholesterol by inhibiting intestinal ACAT activity in hamsters," Evidence-Based Complementary and Alternative Medicine, vol. 2011, Article ID 801272, 9 pages, 2011.

[14] D.-H. Kuo, C.-H. Yeh, P.-C. Shieh, K.-C. Cheng, F.-A. Chen, and J.-T. Cheng, "Effect of ShanZha, a Chinese herbal product, on obesity and dyslipidemia in hamsters receiving high-fat diet," Journal of Ethnopharmacology, vol. 124, no. 3, pp. 544-550, 2009.

[15] C.-S. Niu, C.-T. Chen, L.-J. Chen, K.-C. Cheng, C.-H. Yeh, and J.-T. Cheng, "Decrease of blood lipids induced by Shan-Zha (fruit of Crataegus pinnatifida) is mainly related to an increase of PPAR $\alpha$ in liver of mice fed high-fat diet," Hormone and Metabolic Research, vol. 43, no. 9, pp. 625-630, 2011.
[16] T. Bahorun, F. Trotin, J. Pommery, J. Vasseur, and M. Pinkas, "Antioxidant activities of Crataegus monogyna extracts," Planta Medica, vol. 60, no. 4, pp. 323-328, 1994.

[17] J. Vibes, B. Lasserre, J. Gleye, and C. Declume, "Inhibition of thromboxane $\mathrm{A}_{2}$ biosynthesis in vitro by the main components of Crataegus oxyacantha (Hawthorn) flower heads," Prostaglandins Leukotrienes and Essential Fatty Acids, vol. 50, no. 4, pp. 173-175, 1994.

[18] J. Zand, F. Lanza, H. K. Garg, and N. S. Bryan, "All-natural nitrite and nitrate containing dietary supplement promotes nitric oxide production and reduces triglycerides in humans," Nutrition Research, vol. 31, no. 4, pp. 262-269, 2011.

[19] Z. Zhang, W. K. Ho, Y. Huang, E. J. Anthony, L. W. Lam, and Z.-Y. Chen, "Hawthorn fruit is hypolipidemic in rabbits fed a high cholesterol diet," The Journal of Nutrition, vol. 132, no. 1, pp. 5-10, 2002.

[20] H. Xu, H.-E. Xu, and D. Ryan, "A study of the comparative effects of Hawthorn fruit compound and Simvastatin on lowering blood lipid levels," American Journal of Chinese Medicine, vol. 37, no. 5, pp. 903-908, 2009.

[21] T. Wang, Y. An, C. Zhao et al., "Regulation effects of Crataegus pinnatifida leaf on glucose and lipids metabolism," Journal of Agricultural and Food Chemistry, vol. 59, no. 9, pp. 4987-4994, 2011.

[22] J. D. Chen, Y. Z. Wu, Z. L. Tao, Z. M. Chen, and X. P. Liu, "Hawthorn (shan zha) drink and its lowering effect on blood lipid levels in humans and rats," World Review of Nutrition and Dietetics, vol. 77, pp. 147-154, 1995.

[23] Q.-L. Liu, J.-H. Xiao, R. Ma, Y. Ban, and J.-L. Wang, "Effect of 2,3,5,4'-tetrahydroxystilbene-2-O-beta-d-glucoside on lipoprotein oxidation and proliferation of coronary arterial smooth cells," Journal of Asian Natural Products Research, vol. 9, no. 8, pp. 689-697, 2007.

[24] H.-R. Lin, "Triterpenes from Alisma orientalis act as farnesoid $\mathrm{X}$ receptor agonists," Bioorganic \& Medicinal Chemistry Letters, vol. 22, no. 14, pp. 4787-4792, 2012.

[25] X. Hong, H. Tang, L. Wu, and A. Li, "Protective effects of the Alisma orientalis extract on the experimental nonalcoholic fatty liver disease," Journal of Pharmacy and Pharmacology, vol. 58, no. 10, pp. 1391-1398, 2006.

[26] H. J. Wang and B. H. Chiang, "Anti-diabetic effect of a traditional Chinese medicine formula," Food \& Function, vol. 3, no. 11, pp. 1161-1169, 2012.

[27] B. S. Sanodiya, G. S. Thakur, R. K. Baghel, G. B. K. S. Prasad, and P. S. Bisen, "Ganoderma lucidum: a potent pharmacological macrofungus," Current Pharmaceutical Biotechnology, vol. 10, no. 8, pp. 717-742, 2009.

[28] K. Hasanudin, P. Hashim, and S. Mustafa, "Corn silk (Stigma maydis) in healthcare: a phytochemical and pharmacological review," Molecules, vol. 17, no. 8, pp. 9697-9715, 2012.

[29] S. W. Seto, T. Y. Lam, H. L. Tam et al., "Novel hypoglycemic effects of Ganoderma lucidum water-extract in obese/diabetic $(+d b /+d b)$ mice," Phytomedicine, vol. 16, no. 5, pp. 426-436, 2009.

[30] T. T. Chu, I. F. Benzie, C. W. Lam, B. S. Fok, K. K. Lee, and B. Tomlinson, "Study of potential cardioprotective effects of Ganoderma lucidum (Lingzhi): results of a controlled human intervention trial," British Journal of Nutrition, vol. 107, no. 7, pp. 1017-1027, 2012.

[31] S. Wachtel-Galor, B. Tomlinson, and I. F. F. Benzie, "Ganoderma lucidum ("Lingzhi"), a Chinese medicinal mushroom: 
biomarker responses in a controlled human supplementation study," British Journal of Nutrition, vol. 91, no. 2, pp. 263-269, 2004.

[32] S. R. Kaup, N. Arunkumar, L. K. Bernhardt et al., "Antihyperlipedemic activity of Cynodon dactylon extract in highcholesterol diet fed Wistar rats," Genomic Medicine, Biomarkers, and Health Sciences, vol. 3, no. 3, pp. 98-102, 2011.

[33] Y. Kobayashi, M. Miyazawa, A. Kamei, K. Abe, and T. Kojima, "Ameliorative effects of mulberry (Morus alba L.) leaves on hyperlipidemia in rats fed a high-fat diet: induction of fatty acid oxidation, inhibition of lipogenesis, and suppression of oxidative stress," Bioscience, Biotechnology and Biochemistry, vol. 74, no. 12, pp. 2385-2395, 2010.

[34] Y. J. Lee, D. H. Choi, E. J. Kim et al., "Hypotensive, hypolipidemic, and vascular protective effects of Morus alba L. in rats fed an atherogenic diet," The American Journal of Chinese Medicine, vol. 39, no. 1, pp. 39-52, 2011.

[35] L. I.-K. Liu, F.-P. I. Chou, Y. I.-C. Chen, C.-C. Chyau, H.-H. Ho, and C.-J. Wang, "Effects of mulberry (Morus alba L.) extracts on lipid homeostasis in vitro and in vivo," Journal of Agricultural and Food Chemistry, vol. 57, no. 16, pp. 7605-7611, 2009.

[36] E. Dalli, E. Colomer, M. C. Tormos et al., "Crataegus laevigata decreases neutrophil elastase and has hypolipidemic effect: a randomized, double-blind, placebo-controlled trial," Phytomedicine, vol. 18, no. 8-9, pp. 769-775, 2011.

[37] W. T. Friedewald, R. I. Levy, and D. S. Fredrickson, "Estimation of the concentration of low-density lipoprotein cholesterol in plasma, without use of the preparative ultracentrifuge," Clinical Chemistry, vol. 18, no. 6, pp. 499-502, 1972.

[38] S. Sun, H. Xu, and L. Ngeh, "The evaluation of Chinese therapeutic food for the treatment of moderate dyslipidemia," Evidence-Based Complementary and Alternative Medicine, vol. 2012, Article ID 508683, 10 pages, 2012. 


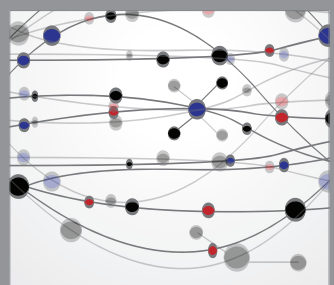

The Scientific World Journal
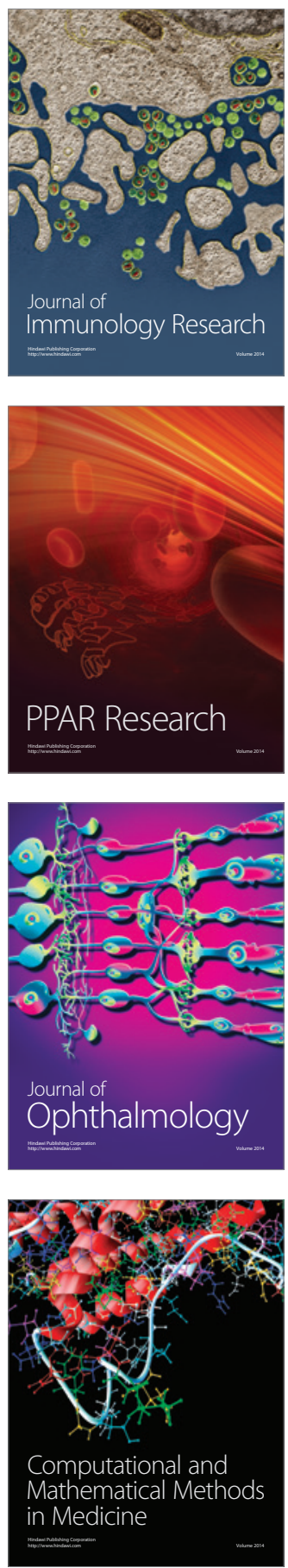

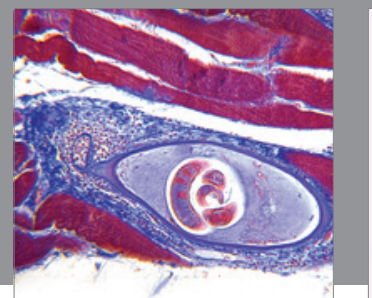

Gastroenterology

Research and Practice
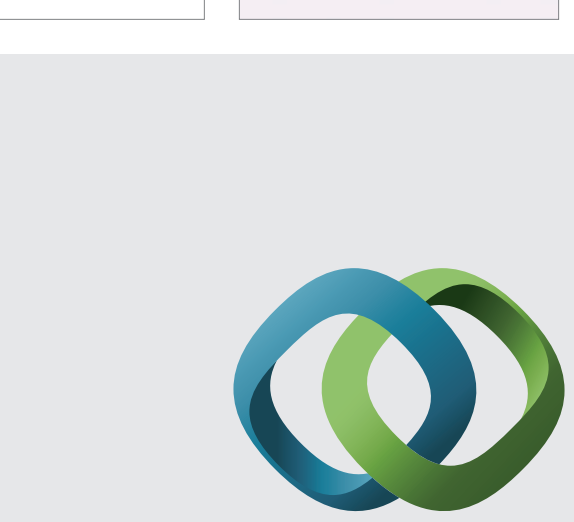

\section{Hindawi}

Submit your manuscripts at

http://www.hindawi.com
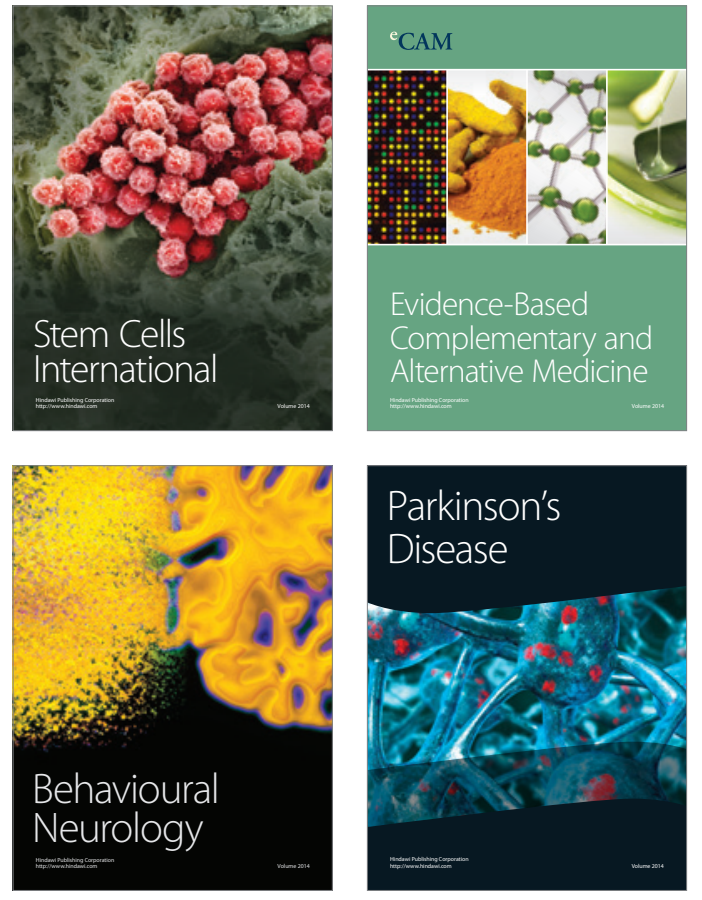
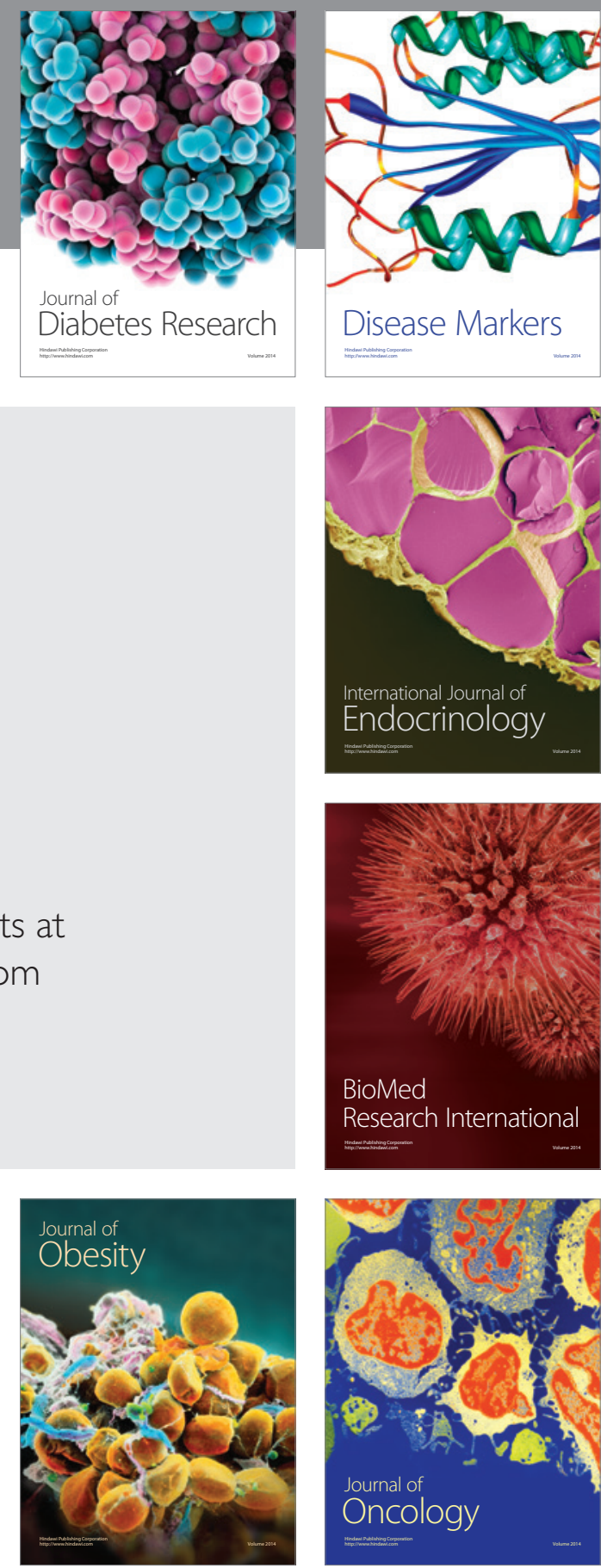

Disease Markers
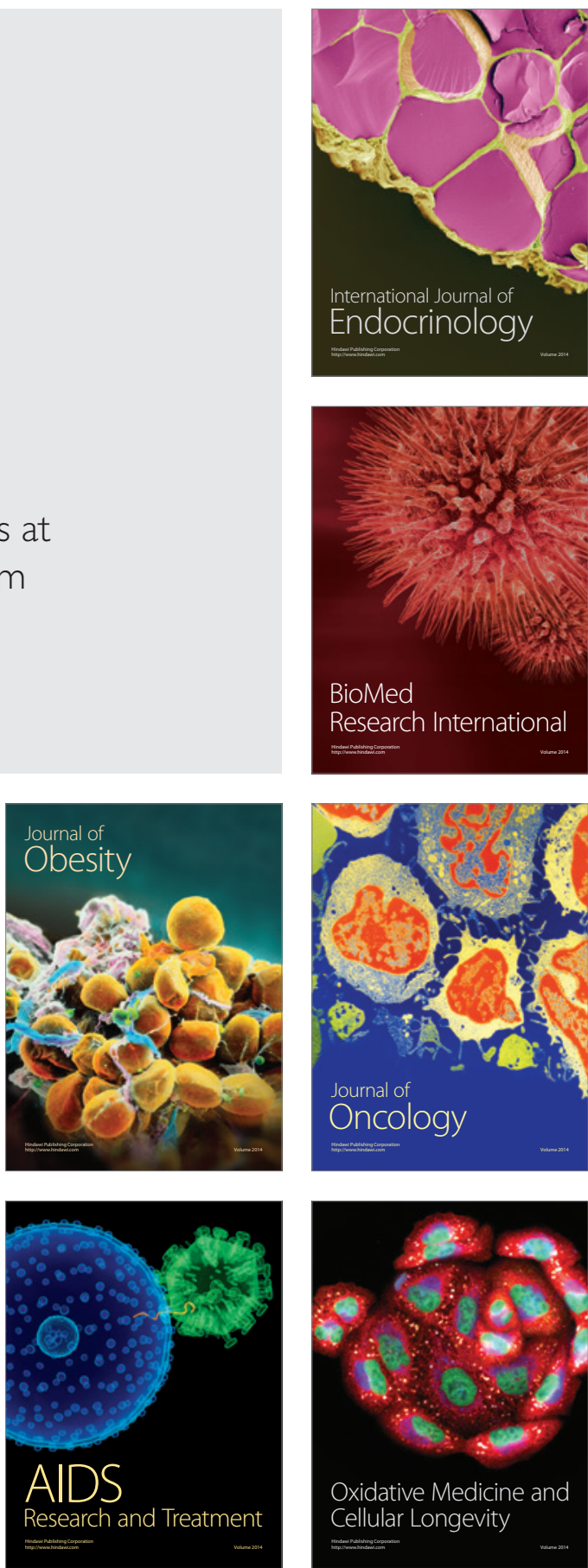